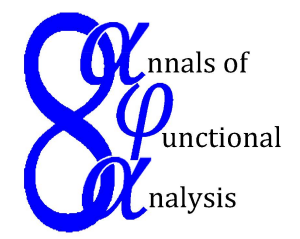

Ann. Funct. Anal. 6 (2015), no. 1, 116-138

http://doi.org/10.15352/afa/06-1-10

ISSN: 2008-8752 (electronic)

http://projecteuclid.org/afa

\title{
EXISTENCE OF STEPANOV-LIKE SQUARE-MEAN PSEUDO ALMOST PERIODIC SOLUTIONS TO PARTIAL STOCHASTIC NEUTRAL DIFFERENTIAL EQUATIONS
}

\author{
ZUOMAO YAN ${ }^{1 *}$ AND HONGWU ZHANG ${ }^{2}$ \\ Communicated by C. Cuevas
}

\begin{abstract}
In this paper, we introduce the concept of Stepanov-like squaremean pseudo almost periodic functions and establish the existence and uniqueness of square-mean almost periodic mild solutions for several neutral partial stochastic differential equations with Stepanov-like almost periodic coefficients in a real separable Hilbert space. Moreover, two examples are given to illustrate the general theorems.
\end{abstract}

\section{INTRODUCTION}

The theory of neutral differential equations arises in many phenomena such as in the study of oscillatory systems and also in the modeling of several physical problems. It has been widely studied in the last decades. There exists an extensive literature for abstract partial neutral differential equations we refer to the papers [2],[22],[23],[33],[34], the book [32]. The existence of almost periodic, asymptotically almost periodic, and pseudo-almost periodic solutions are the most attractive topics in qualitative theories of differential equations because of their significance and applications in physics, mechanics and mathematical biology. The concept of pseudo almost periodicity was introduced by Zhang in [35],[36],[37],[38] in the early nineties. In recent years, the existence of pseudo-almost periodic solutions on different kinds of differential equations have been considered in many publications such as [3], [11],[12],[13],[14],[17],[18],[19],[20],[24],[25],[39]. and the reference there in. Especially, Diagana has, in [15],[16], introduced the notion of

Date: Received: Jul. 17, 2013; Accepted: Sep. 31, 2013.

* Corresponding author.

2010 Mathematics Subject Classification. Primary 34K14; Secondary 35B15, 34F05, 60H10.

Key words and phrases. Square-mean pseudo almost periodic, Stepanov-like pseudo almost periodic, Neutral partial stochastic differential equations, Exponentially stable semigroup. 
Stepanov-like pseudo almost periodicity as a natural generalization of the concept of pseudo almost periodicity as well as the one of Stepanov-like almost periodicity, established a composition theorem on Stepanov-like pseudo almost periodic functions, and [26] for more recent results about the Stepanov-like almost periodic function theory.

Recently, there has been an increasing interest in extending certain classical deterministic results to stochastic differential equations. This is due to the fact that most problems in a real life situation to which mathematical models are applicable are basically stochastic rather than deterministic. The existence of almost periodic, asymptotically almost periodic, and pseudo almost periodic solutions to some stochastic differential equations has been considered in many publications such as [4],[29],[21],[30],[31],[10],[27],[5], [6],[7],[8],[9] and references therein. Among them, Tudor [30] studied the almost periodic solution for the affine and stochastic evolution equations. In [31], the pseudo almost periodic solution for a class of stochastic differential equations was investigated. Cao et al.[10] proved the existence and exponential stability of quadratic-mean asymptotically almost periodic mild solutions to a class of stochastic differential equations. Huang and Yang [27] presented some new criteria ensuring the existence and uniqueness of quadratic-mean almost periodic solution as well as global exponential stability of the almost periodic solution for stochastic cellular neural networks with delay. Bezandry and Diagana [5] considered the almost periodic solution for a class of stochastic differential equations. In [6],[7],[8], the existence of square-mean almost periodic solutions to some stochastic differential equations on a Hilbert space was investigated. In particular, Bezandry and Diagana [9] studied the notion of Stepanov almost periodicity (or $S^{2}$-almost periodicity) for stochastic processes, which is weaker than the notion of quadratic-mean almost periodicity. They results are more general and complicated than the almost periodic solutions or pseudo almost periodic solutions to some stochastic differential equations.

In this paper, we investigate the existence and uniqueness of Stepanov-like square-mean pseudo almost periodic mild solutions to the following neutral partial stochastic differential equations:

$$
\begin{gathered}
d x(t)=A x(t) d t+d g(t, x(t))+h(t, x(t)) d t+f(t, x(t)) d W(t), \quad t \in \mathbb{R}, \quad(1.1) \\
d x(t)=A x(t) d t+d g\left(t, B_{1} x(t)\right)+h\left(t, B_{2} x(t)\right) d t+f\left(t, B_{3} x(t)\right) d W(t), \quad t \in \mathbb{R},
\end{gathered}
$$

where $A$ is the infinitesimal generator of a $C_{0}$-semigroup $\{T(t)\}_{t \geq 0}$ on $L^{2}(\mathbb{P}, \mathbb{H})$ and $W(t)$ is a two-sided standard one-dimensional Brownian motion defined on the filtered probability space $\left(\Omega, \mathcal{F}, \mathbb{P}, \mathcal{F}_{t}\right)$, where $\mathcal{F}_{t}=\sigma\{W(u)-W(v) ; u, v \leq t\}$. $g, h, f, g$ and $B_{i}, i=1,2,3$, are appropriate functions to be specified later.

Existence results related to almost periodic solutions to abstract partial neutral differential equations have recently been established in [20],[23],[39],[1],[25], respectively. However, the existence of square-mean pseudo almost periodic solutions to neutral partial stochastic differential equations of the form (1.1) and 
(1.2) in the case when the forcing terms $g, h, f$ are Stepanov-like almost periodic coefficients is an untreated topic and constitutes the main motivation of the present paper. For this reason, we introduce and study the notion of Stepanovlike square-mean pseudo almost periodic for stochastic processes, which, in turn generalizes all the above-mentioned concepts, in particular, the notion of squaremean pseudo almost periodic. As an application, we study and obtain the existence and uniqueness of square-mean pseudo almost periodic mild solutions to a neutral stochastic functional differential equations with Stepanov-like-pseudo almost automorphic coefficients. Such a result generalizes most of known results on the existence of almost periodic (respectively, pseudo almost periodic) solutions to stochastic differential equations of type Eq. (1.1) and Eq. (1.2).

The paper is organized as follows. In Section 2, we recall briefly some basic notations and definitions, lemmas related with square-mean pseudo almost periodic functions and Stepanov-like square-mean pseudo almost periodic functions. Section 3 verifies the existence and uniqueness of the Stepanov-like square-mean pseudo almost periodic solutions for the problems (1.1) and (1.2). Finally in Section 4, we investigate two examples to illustrate the abstract results.

\section{PRELIMINARIES}

In this section, we introduce some basic definitions, notations and lemmas which are used throughout this paper.

Throughout the paper,we assume that $(\mathbb{H},\|\cdot\|,\langle\cdot, \cdot\rangle)$ and $\left(\mathbb{K},\|\cdot\|_{\mathbb{K}},\langle\cdot, \cdot\rangle_{\mathbb{K}}\right)$ are two real separable Hilbert spaces. Let $(\Omega, \mathcal{F}, \mathbb{P})$ be a complete probability space. The notation $L^{2}(\mathbb{P}, \mathbb{H})$ stands for the space of all $\mathbb{H}$-valued random variables $x$ such that $E\|x\|^{2}=\int_{\Omega}\|x\|^{2} d \mathbb{P}<\infty$, which is a Banach space with the norm $\|x\|_{2}=\left(\int_{\Omega}\|x\|^{2} d \mathbb{P}\right)^{\frac{1}{2}}$. It is routine to check that $L^{2}(\mathbb{P}, \mathbb{H})$ is a Hilbert space equipped with the norm $\|\cdot\|$. We let $L(\mathbb{K}, \mathbb{H})$ be the space of all linear bounded operators from $\mathbb{K}$ into $\mathbb{H}$, equipped with the usual operator norm $\|\cdot\|_{L(\mathbb{K}, \mathbb{H})}$; in particular, this is simply denoted by $L(\mathbb{H})$ when $\mathbb{K}=\mathbb{H}$. $W(t)$ is a two-sided standard one-dimensional Brownian motion defined on the filtered probability space $\left(\Omega, \mathcal{F}, \mathbb{P}, \mathcal{F}_{t}\right)$, where $\mathcal{F}_{t}=\sigma\{W(u)-W(v) ; u, v \leq t\}$.

In this paper, the operator $A$ is the infinitesimal generator of an exponentially stable $C_{0}$-semigroup $\{T(t)\}_{t \geq 0}$ on $L^{2}(\mathbb{P}, \mathbb{H})$; that is, there exist $M>0, \delta>0$ such that

$$
\|T(t)\| \leq M e^{-\delta t}
$$

for all $t \geq 0$.

2.1. Square-mean Pseudo almost periodicity. The notations $C\left(\mathbb{R}, L^{2}(\mathbb{P}, \mathbb{H})\right)$, and $B C\left(\mathbb{R}, L^{2}(\mathbb{P}, \mathbb{H})\right)$ stand for the collection of all continuous functions from $\mathbb{R}$ into $L^{2}(\mathbb{P}, \mathbb{H})$, the Banach space of all bounded continuous functions from $\mathbb{R}$ into $L^{2}(\mathbb{P}, \mathbb{H})$, equipped with the sup norm $\|\cdot\|_{\infty}$, respectively. Let $B C\left(\mathbb{R}, L^{2}(\mathbb{P}, \mathbb{K})\right)$ denote the Banach space of all bounded continuous functions from $\mathbb{R}$ to $L^{2}(\mathbb{P}, \mathbb{K})$, equipped with the $L^{2}(\mathbb{P}, \mathbb{K})$-sup norm $\|\cdot\|_{\infty, \mathbb{K}}$.

Similarly, $C\left(\mathbb{R} \times L^{2}(\mathbb{P}, \mathbb{K}), L^{2}(\mathbb{P}, \mathbb{H})\right)$ and $B C\left(\mathbb{R} \times L^{2}(\mathbb{P}, \mathbb{K}), L^{2}(\mathbb{P}, \mathbb{H})\right)$ stand, respectively, for the class of all jointly continuous functions from $\mathbb{R} \times L^{2}(\mathbb{P}, \mathbb{K})$ 
into $L^{2}(\mathbb{P}, \mathbb{H})$ and the collection of all jointly bounded continuous functions from $\mathbb{R} \times L^{2}(\mathbb{P}, \mathbb{K})$ into $L^{2}(\mathbb{P}, \mathbb{H})$.

Definition 2.1. A stochastic process $x(t): \mathbb{R} \rightarrow L^{2}(\mathbb{P}, \mathbb{H})$ is said to be stochastically bounded if there exists $\widehat{M}>0$ such that $E\|x(t)\|^{2} \leq \widehat{M}$ for all $t \in \mathbb{R}$.

Definition 2.2. A stochastic process $x: \mathbb{R} \rightarrow L^{2}(\mathbb{P}, \mathbb{H})$ is said to be stochastically continuous if

$$
\lim _{t \rightarrow s} E\|x(t)-x(s)\|^{2}=0 .
$$

Denote by $B C\left(\mathbb{R}, L^{2}(\mathbb{P}, \mathbb{H})\right)$ the collection of all the stochastically bounded and continuous processes. Then several properties of the space $B C\left(\mathbb{R}, L^{2}(\mathbb{P}, \mathbb{H})\right)$ are listed as follows.

Remark 2.3. $B C\left(\mathbb{R}, L^{2}(\mathbb{P}, \mathbb{H})\right)$ is a linear space.

Remark 2.4. $B C\left(\mathbb{R}, L^{2}(\mathbb{P}, \mathbb{H})\right)$ is a Banach space with the norm

$$
\|x\|_{\infty}:=\sup _{t \in \mathbb{R}}\left(E\|x(t)\|^{2}\right)^{\frac{1}{2}}
$$

for $E\|x(t)\|^{2}=\left(\int_{\Omega}\|x(t)\|^{2} d \mathbb{P}\right)^{\frac{1}{2}}$.

Definition 2.5. ([6],[7],[8]) A stochastic process $x \in C\left(\mathbb{R}, L^{2}(\mathbb{P}, \mathbb{H})\right)$ is called (Bohr) square-mean almost periodic if for each $\varepsilon>0$ there exists $l(\varepsilon)>0$ such that every interval of length $l(\varepsilon)$ contains a number $s^{\prime}$ with the property that

$$
E\left\|x\left(t+s^{\prime}\right)-x(t)\right\|^{2}<\varepsilon
$$

for each $t \in \mathbb{R}$. The collection of all such functions will be denoted by $A P\left(L^{2}(\mathbb{P}, \mathbb{H})\right)$.

Denote

$$
P A P_{0}\left(\mathbb{R}, L^{2}(\mathbb{P}, \mathbb{H})\right)=\left\{f \in B C\left(\mathbb{R}, L^{2}(\mathbb{P}, \mathbb{H})\right): \lim _{r \rightarrow \infty} \frac{1}{2 r} \int_{-r}^{r} E\|f(t)\|^{2} d t=0\right\} .
$$

Definition 2.6. (Compare with [31]) A stochastic process $f \in B C\left(\mathbb{R}, L^{2}(\mathbb{P}, \mathbb{H})\right.$ ) is said to be square-mean pseudo almost periodic if it can be decomposed as $f=g+\varphi$, where $g \in A P\left(L^{2}(\mathbb{P}, \mathbb{H})\right)$ and $\varphi \in P A P_{0}\left(L^{2}(\mathbb{P}, \mathbb{H})\right)$. The collection of such functions will be denoted by $P A P\left(L^{2}(\mathbb{P}, \mathbb{H})\right)$.

We define $P A P_{0}\left(\mathbb{R}, L^{2}(\mathbb{P}, \mathbb{K})\right)$ as the collection of functions $f \in B C(\mathbb{R} \times$ $\left.L^{2}(\mathbb{P}, \mathbb{K}), L^{2}(\mathbb{P}, \mathbb{H})\right)$ such that

$$
\lim _{r \rightarrow \infty} \frac{1}{2 r} \int_{-r}^{r} E\|f(t, x)\|^{2} d t=0
$$

uniformly in $x \in L^{2}(\mathbb{P}, \mathbb{K})$.

Definition 2.7. ([6],[7],[8]) A stochastic process $f \in C\left(\mathbb{R} \times L^{2}(\mathbb{P}, \mathbb{K}), L^{2}(\mathbb{P}, \mathbb{H})\right)$ is called (Bohr) almost periodic in $t \in \mathbb{R}$ uniformly in $x \in L^{2}(\mathbb{P}, \mathbb{K})$ ) if for each $\varepsilon>0$ and any compact $K \subset L^{2}(\mathbb{P}, \mathbb{K})$ there exists $l(\varepsilon)>0$ such that every interval of length $l(\varepsilon)$ contains a number $s^{\prime}$ with the property that

$$
E\left\|f\left(t+s^{\prime}, x\right)-f(t, x)\right\|^{2}<\varepsilon
$$

for each $t \in \mathbb{R}, x \in K$. The collection of all such functions will be denoted by $A P\left(\mathbb{R} \times L^{2}(\mathbb{P}, \mathbb{K})\right)$. 
Lemma 2.8. Assume $\left\{f_{n}\right\}_{n \in \mathbb{N}} \subset P A P\left(L^{2}(\mathbb{P}, \mathbb{H})\right)$ be a sequence of stochastic processes. If $\left\{f_{n}\right\}$ converges uniformly to some $f$, then $f \in P A P\left(L^{2}(\mathbb{P}, \mathbb{H})\right)$.

One can refer to Lemma 2.5 in [16] for the proof of Lemma 2.8.

Definition 2.9. ([31]) A stochastic process $f \in C\left(\mathbb{R} \times L^{2}(\mathbb{P}, \mathbb{K}), L^{2}(\mathbb{P}, \mathbb{H})\right)$ is said to be pseudo almost periodic in $t \in \mathbb{R}$ uniformly in $u \in L^{2}(\mathbb{P}, \mathbb{K})$ if it can be decomposed as $f=g+\varphi$, where $g \in A P\left(\mathbb{R} \times L^{2}(\mathbb{P}, \mathbb{K})\right)$ and $\varphi \in P A P_{0}(\mathbb{R} \times$ $\left.L^{2}(\mathbb{P}, \mathbb{K})\right)$. The collection of such functions will be denoted by $P A P\left(\mathbb{R} \times L^{2}(\mathbb{P}, \mathbb{K})\right)$.

Lemma 2.10. ([31]) Assume $f \in P A P\left(\mathbb{R} \times L^{2}(\mathbb{P}, \mathbb{K})\right)$. Suppose that $f(t, u)$ is Lipschitz in $u \in L^{2}(\mathbb{P}, \mathbb{H})$ uniformly in $t \in \mathbb{R}$, in the sense that there exists $L^{*}>0$ such that

$$
E\|f(t, u)-f(t, v)\|^{2} \leq L^{*} E\|u-v\|^{2}
$$

for all $t \in \mathbb{R}, u, v \in L^{2}(\mathbb{P}, \mathbb{K})$.

If $\phi(\cdot) \in P A P\left(L^{2}(\mathbb{P}, \mathbb{K})\right)$ then $f(\cdot, \phi(\cdot)) \in P A P\left(L^{2}(\mathbb{P}, \mathbb{H})\right)$.

Lemma 2.11. Let $u \in P A P\left(L^{2}(\mathbb{P}, \mathbb{K})\right), B \in L\left(L^{2}(\mathbb{P}, \mathbb{K}), L^{2}(\mathbb{P}, \mathbb{H})\right)$. If $v(t)=$ $B u(t)$ for each $t \in \mathbb{R}$, then $v \in P A P\left(L^{2}(\mathbb{P}, \mathbb{H})\right)$.

One can refer to Lemma 4.1 in [26] for the proof of Lemma 2.11.

\subsection{Stepanov-like square-mean pseudo almost periodicity.}

Definition 2.12. ([9]) The Bochner transform $x^{b}(t, s), t \in \mathbb{R}, s \in[0,1]$, of a stochastic process $x: \mathbb{R} \rightarrow L^{2}(\mathbb{P}, \mathbb{H})$ is defined by

$$
x^{b}(t, s):=x(t+s) .
$$

Remark 2.13. ([9]) A stochastic process $\psi(t, s), t \in \mathbb{R}, s \in[0,1]$, is the Bochner transform of a certain stochastic process $x$,

$$
\psi(t, s)=x^{b}(t, s),
$$

if and only if

$$
\psi(t+\tau, s-\tau)=\psi(s, t)
$$

for all $t \in \mathbb{R}, s \in[0,1]$ and $\tau \in[s-1, s]$.

Definition 2.14. ([9]) The Bochner transform $F^{b}(t, s, u), t \in \mathbb{R}, s \in[0,1], u \in$ $L^{2}(\mathbb{P}, \mathbb{H})$, of a function $F: \mathbb{R} \times L^{2}(\mathbb{P}, \mathbb{H}) \rightarrow L^{2}(\mathbb{P}, \mathbb{H})$ is defined by

$$
F^{b}(t, s, u):=F(t+s, u)
$$

for each $u \in L^{2}(\mathbb{P}, \mathbb{H})$.

Definition 2.15. ([9]) The space $B S^{2}\left(L^{2}(\mathbb{P}, \mathbb{H})\right)$ of all Stepanov bounded stochastic processes consists of all measurable stochastic processes $x: \mathbb{R} \rightarrow L^{2}(\mathbb{P}, \mathbb{H})$ such that

$$
x^{b} \in L^{\infty}\left(\mathbb{R}, L^{2}\left(0,1 ; L^{2}(\mathbb{P}, \mathbb{H})\right)\right) .
$$

This is a Banach space with the norm

$$
\|x\|_{S^{2}}=\left\|x^{b}\right\|_{L^{\infty}\left(\mathbb{R}, L^{2}\right)}=\sup _{t \in \mathbb{R}}\left(\int_{t}^{t+1} E\|x(\tau)\|^{2} d \tau\right)^{\frac{1}{2}} .
$$


Definition 2.16. A stochastic process $f \in B S^{2}\left(\mathbb{R}, L^{2}(\mathbb{P}, \mathbb{H})\right)$ is said to be Stepanovlike square-mean pseudo almost periodic (or $S^{2}$-pseudo almost periodic) if it can be decomposed as $f=h+\varphi$, where $h^{b} \in A P\left(L^{2}\left(0,1 ; L^{2}(\mathbb{P}, \mathbb{H})\right)\right)$ and $\varphi^{b} \in$ $P A P_{0}\left(L^{2}\left(0,1 ; L^{2}(\mathbb{P}, \mathbb{H})\right)\right)$. Denote the set of all such stochastically continuous processes by $P A P S^{2}\left(L^{2}(\mathbb{P}, \mathbb{H})\right)$.

In other words, a stochastic process $f \in L_{\text {loc }}^{2}\left(\mathbb{R}, L^{2}(\mathbb{P}, \mathbb{H})\right)$ is said to be Stepanovlike square-mean pseudo almost periodic if its Bochner transform $f^{b}: \mathbb{R} \rightarrow$ $L^{2}\left(0,1 ; L^{2}(\mathbb{P}, \mathbb{H})\right)$ is square-mean pseudo almost automorphic in the sense that there exist two functions $h, \varphi: \mathbb{R} \rightarrow L^{2}(\mathbb{P}, \mathbb{H})$ such that $f=h+\varphi$, where $h^{b} \in A P\left(L^{2}\left(0,1 ; L^{2}(\mathbb{P}, \mathbb{H})\right)\right)$ and $\varphi^{b} \in P A P_{0}\left(L^{2}\left(0,1 ; L^{2}(\mathbb{P}, \mathbb{H})\right)\right)$.

Obviously, the following inclusions hold:

$$
A P\left(L^{2}(\mathbb{P}, \mathbb{H})\right) \subset P A P\left(L^{2}(\mathbb{P}, \mathbb{H})\right) \subset P A P S^{2}\left(L^{2}(\mathbb{P}, \mathbb{H})\right)
$$

Definition 2.17. A stochastic process $f \in B S^{2}\left(\mathbb{R} \times L^{2}(\mathbb{P}, \mathbb{K}), L^{2}(\mathbb{P}, \mathbb{H})\right)$ is said to be Stepanov-like square-mean pseudo almost periodic (or $S^{2}$-pseudo almost periodic) if it can be decomposed as $f=h+\varphi$, where $h^{b} \in A P\left(\mathbb{R} \times L^{2}\left(0,1 ; L^{2}(\mathbb{P}, \mathbb{K})\right)\right)$ and $\varphi^{b} \in P A P_{0}\left(\mathbb{R} \times L^{2}\left(0,1 ; L^{2}(\mathbb{P}, \mathbb{K})\right)\right)$. Denote the set of all such stochastically continuous processes by $P A P S^{2}\left(\mathbb{R} \times L^{2}(\mathbb{P}, \mathbb{K})\right)$.

Lemma 2.18. Assume $f \in P A P S^{2}\left(\mathbb{R} \times L^{2}(\mathbb{P}, \mathbb{K})\right)$. Suppose that $f(t, u)$ is Lipschitz in $u \in L^{2}(\mathbb{P}, \mathbb{K})$ uniformly in $t \in \mathbb{R}$, in the sense that there exists $\tilde{L}>0$ such that

$$
E\|f(t, u)-f(t, v)\|^{2} \leq \tilde{L} E\|u-v\|^{2}
$$

for all $t \in \mathbb{R}, u, v \in L^{2}(\mathbb{P}, \mathbb{K})$. If $\phi(\cdot) \in \operatorname{PAPS}^{2}\left(L^{2}(\mathbb{P}, \mathbb{K})\right)$ then $f(\cdot, \phi(\cdot)) \in$ $\operatorname{PAPS}^{2}\left(L^{2}(\mathbb{P}, \mathbb{H})\right)$.

Lemma 2.18 can be proved by using Definition 2.16, Definition 2.17 and Lemmas 2.10. One may refer to Theorem 2.14 in [15] for more details about the proof of Lemma 2.18.

Lemma 2.19. Let $x(\cdot) \in P A P S^{2}\left(L^{2}(\mathbb{P}, \mathbb{K})\right), B \in L\left(L^{2}(\mathbb{P}, \mathbb{K}), L^{2}(\mathbb{P}, \mathbb{H})\right)$. If $y(t)=$ $B x(t)$ for each $t \in \mathbb{R}$, then $y \in P A P S^{2}\left(L^{2}(\mathbb{P}, \mathbb{H})\right)$.

The proof is similar to the proof of Theorem 4.2 in [26], and we omit the details here.

\section{Existence of Stepanov-like Square-mean PSEudo Almost Periodic SOLUTIONS}

In this section, we prove that there is a unique mild solution for the problems (1.1) and (1.2). We first prove the next auxiliary results.

Lemma 3.1. If $h \in P A P S^{2}\left(L^{2}(\mathbb{P}, \mathbb{H})\right) \cap C\left(\mathbb{R}, L^{2}(\mathbb{P}, \mathbb{H})\right)$ and if $H$ is the function defined by

$$
H(t):=\int_{-\infty}^{t} T(t-\tau) h(\tau) d \tau
$$

for each $t \in \mathbb{R}$, then $H \in P A P\left(L^{2}(\mathbb{P}, \mathbb{H})\right)$. 
Proof. Since $h \in P A P S^{2}\left(L^{2}(\mathbb{P}, \mathbb{H})\right) \cap C\left(\mathbb{R}, L^{2}(\mathbb{P}, \mathbb{H})\right)$, write

$$
h=h_{1}+h_{2},
$$

where

$$
h_{1}^{b} \in A P\left(L^{2}\left(0,1 ; L^{2}(\mathbb{P}, \mathbb{H})\right)\right) \cap C\left(\mathbb{R}, L^{2}\left(0,1 ; L^{2}(\mathbb{P}, \mathbb{H})\right)\right)
$$

and

$$
h_{2}^{b} \in P A P_{0}\left(L^{2}\left(0,1 ; L^{2}(\mathbb{P}, \mathbb{H})\right)\right) \cap C\left(\mathbb{R}, L^{2}\left(0,1 ; L^{2}(\mathbb{P}, \mathbb{H})\right)\right),
$$

then $H(t)$ can be decomposed as

$$
H(t)=\int_{-\infty}^{t} T(t-\tau) h_{1}(\tau) d \tau+\int_{-\infty}^{t} T(t-\tau) h_{2}(\tau) d \tau
$$

Define for all $k=1,2,3, \ldots$, the sequence of integral operators

$$
H_{k}(t)=\int_{k-1}^{k} T(\tau) h_{1}(t-\tau) d \tau+\int_{k-1}^{k} T(\tau) h_{2}(t-\tau) d \tau .
$$

Set

$$
H_{1, k}(t)=\int_{k-1}^{k} T(\tau) h_{1}(t-\tau) d \tau=\int_{t-k}^{t-k+1} T(t-\tau) h_{1}(\tau) d \tau
$$

and

$$
H_{2, k}(t)=\int_{k-1}^{k} T(\tau) h_{2}(t-\tau) d \tau=\int_{t-k}^{t-k+1} T(t-\tau) h_{2}(\tau) d \tau
$$

for each $t \in \mathbb{R}$ and $k=1,2,3, \ldots$ Next we show that $H_{1, k} \in A P\left(L^{2}(\mathbb{P}, \mathbb{H})\right)$ and $H_{2, k} \in P A P_{0}\left(L^{2}(\mathbb{P}, \mathbb{H})\right)$.

To prove that $H_{1, k} \in A P\left(L^{2}(\mathbb{P}, \mathbb{H})\right)$. Using the exponential stable of $T(t)_{t \geq 0}$ and Hölder's inequality, it follows that

$$
\begin{aligned}
E\left\|H_{1, k}(t)\right\|^{2} & =E\left\|\int_{t-k}^{t-k+1} T(t-\tau) h_{1}(\tau) d \tau\right\|^{2} \\
& \leq E\left(\int_{t-k}^{t-k+1}\|T(t-\tau)\|\left\|h_{1}(\tau)\right\| d \tau\right)^{2} \\
& \leq M^{2} E\left(\int_{t-k}^{t-k+1} e^{-\delta(t-\tau)}\left\|h_{1}(\tau)\right\| d \tau\right)^{2} \\
& \leq M^{2}\left(\int_{t-k}^{t-k+1} e^{-2 \delta(t-\tau)} d \tau\right)\left(\int_{t-k}^{t-k+1} E\left\|h_{1}(\tau)\right\|^{2} d \tau\right) \\
& \leq M^{2}\left(\int_{k-1}^{k} e^{-2 \delta \tau} d \tau\right)\left\|h_{1}\right\|_{S^{2}}^{2} \\
& \leq \frac{M^{2}}{2 \delta}\left(e^{2 \delta}-1\right) e^{-2 \delta k}\left\|h_{1}\right\|_{S^{2}}^{2} .
\end{aligned}
$$

Since the series

$$
\frac{M^{2}}{2 \delta}\left(e^{2 \delta}-1\right)\left\|h_{1}\right\|_{S^{2}}^{2} \sum_{k=1}^{\infty} e^{-2 \delta k}<\infty
$$


we deduce from the well-known Weirstrass test that the series $\sum_{k=1}^{\infty} H_{1, k}(t)$ is uniformly convergent on $\mathbb{R}$. Now let

$$
H_{1}(t):=\sum_{k=1}^{\infty} H_{1, k}(t), \quad t \in \mathbb{R} .
$$

Observe that

$$
H_{1}(t):=\int_{-\infty}^{t} T(t-\tau) h_{1}(\tau) d \tau, \quad t \in \mathbb{R}
$$

and hence $H_{1}(t) \in C\left(\mathbb{R}, L^{2}(\mathbb{P}, \mathbb{H})\right)$. Moreover, for any $t \in \mathbb{R}$, we have

$$
\begin{aligned}
E\left\|H_{1}(t)\right\|^{2} & \leq 2 E\left\|H_{1}(t)-\sum_{k=1}^{n} H_{1, k}(t)\right\|^{2}+2\left(\sum_{k=1}^{n} e^{-\delta k}\right)\left(\sum_{k=1}^{n} e^{\delta k} E\left\|H_{1, k}(t)\right\|^{2}\right) \\
& \leq C_{1}(M, \delta)\left\|h_{1}\right\|_{S^{2}}^{2},
\end{aligned}
$$

where $C_{1}(M, \delta)$ depends only on the fixed constants $M$ and $\delta$.

Let us show that each $H_{1, k} \in A P\left(L^{2}(\mathbb{P}, \mathbb{H})\right)$.

Indeed, by $h_{1}^{b} \in A P\left(L^{2}\left(0,1 ; L^{2}(\mathbb{P}, \mathbb{H})\right)\right)$, given $\varepsilon>0$, one can find $l(\varepsilon)>0$ such that any interval of length $l(\varepsilon)$ contains at least $s^{\prime}$ with the property that

$$
\int_{t}^{t+1} E\left\|h_{1}\left(s+s^{\prime}\right)-h_{1}(s)\right\|^{2} d s<\varepsilon
$$

for all $t \in \mathbb{R}$. On the other hand, using the inequality (3.2), exponential stable of $T(t)_{t \geq 0}$ and Hölder's inequality, we obtain that

$$
\begin{aligned}
E & \left\|H_{1, k}\left(t+s^{\prime}\right)-H_{1, k}(t)\right\|^{2} \\
& \leq E\left\|\int_{k-1}^{k} T(\tau)\left[h_{1}\left(t+s^{\prime}-\tau\right)-h_{1}(t-\tau)\right] d \tau\right\|^{2} \\
& \leq E\left(\int_{k-1}^{k}\|T(\tau)\|\left\|h_{1}\left(t+s^{\prime}-\tau\right)-h_{1}(t-\tau)\right\| d \tau\right)^{2} \\
& \leq M^{2} E\left(\int_{k-1}^{k} e^{-\delta \tau}\left\|h_{1}\left(t+s^{\prime}-\tau\right)-h_{1}(t-\tau)\right\| d \tau\right)^{2} \\
& \leq M^{2}\left(\int_{k-1}^{k} e^{-2 \delta \tau} d \tau\right)\left(\int_{k-1}^{k} E\left\|h_{1}\left(t+s^{\prime}-\tau\right)-h_{1}(t-\tau)\right\|^{2} d \tau\right) \\
& \leq \frac{M^{2}}{2 \delta} e^{-2 \delta k}\left(e^{2 \delta}-1\right)\left(\int_{t-k}^{t-k+1} E\left\|h_{1}\left(s+s^{\prime}\right)-h_{1}(s)\right\|^{2} d s\right) \\
& <\frac{M^{2}}{2 \delta} e^{-2 \delta k}\left(e^{2 \delta}-1\right) \varepsilon .
\end{aligned}
$$

Therefore, we deduce that $H_{1, k} \in A P\left(L^{2}(\mathbb{P}, \mathbb{H})\right)$.

Next, we will prove that $H_{2, k} \in P A P_{0}\left(L^{2}(\mathbb{P}, \mathbb{H})\right)$. It is obvious that $H_{2, k} \in$ $B C\left(\mathbb{R}, L^{2}(\mathbb{P}, \mathbb{H})\right)$, the left task is to show that

$$
\lim _{r \rightarrow \infty} \frac{1}{2 r} \int_{-r}^{r} E\left\|H_{2, k}(t)\right\|^{2} d t=0
$$


for each $t \in \mathbb{R}$ and $k=1,2,3, \ldots$ Then, by using the exponential stable of $T(t)_{t \geq 0}$ and Hölder's inequality, it follows that

$$
\begin{aligned}
E\left\|H_{2, k}(t)\right\|^{2} & =E\left\|\int_{t-k}^{t-k+1} T(t-\tau) h_{2}(\tau) d \tau\right\|^{2} \\
& \leq E\left(\int_{t-k}^{t-k+1}\|T(t-\tau)\|\left\|h_{2}(\tau)\right\| d \tau\right)^{2} \\
& \leq E\left(\int_{t-k}^{t-k+1} M e^{-\delta(t-\tau)}\left\|h_{2}(\tau)\right\| d \tau\right)^{2} \\
& \leq M^{2}\left(\int_{t-k}^{t-k+1} e^{-2 \delta(t-\tau)} d \tau\right)\left(\int_{t-k}^{t-k+1} E\left\|h_{2}(\tau)\right\|^{2} d \tau\right) \\
& \leq M^{2}\left(\int_{k-1}^{k} e^{-2 \delta \tau} d \tau\right)\left(\int_{t-k}^{t-k+1} E\left\|h_{2}(\tau)\right\|^{2} d \tau\right) \\
& \leq \frac{M^{2}}{2 \delta} e^{-2 \delta k}\left(e^{2 \delta}-1\right)\left(\int_{t-k}^{t-k+1} E\left\|h_{2}(\tau)\right\|^{2} d \tau\right) \\
& \leq \frac{M^{2}}{2 \delta}\left(e^{2 \delta}-1\right) e^{-2 \delta k}\left\|h_{2}\right\|_{S^{2}}^{2} .
\end{aligned}
$$

Since the series

$$
\frac{M^{2}}{2 \delta}\left(e^{2 \delta}-1\right)\left\|h_{2}\right\|_{S^{2}}^{2} \sum_{k=1}^{\infty} e^{-2 \delta k}<\infty,
$$

we deduce from the well-known Weirstrass test that the series $\sum_{k=1}^{\infty} H_{2, k}(t)$ is uniformly convergent on $\mathbb{R}$. Now let

$$
H_{2}(t):=\sum_{k=1}^{\infty} H_{2, k}(t), \quad t \in \mathbb{R} .
$$

Observe that

$$
H_{2}(t):=\int_{-\infty}^{t} T(t-\tau) h_{2}(\tau) d \tau, \quad t \in \mathbb{R}
$$

and hence $H_{2}(t) \in C\left(\mathbb{R}, L^{2}(\mathbb{P}, \mathbb{H})\right)$. Moreover, for any $t \in \mathbb{R}$, we have

$$
\begin{aligned}
E\left\|H_{2}(t)\right\|^{2} & \leq 2 E\left\|H_{2}(t)-\sum_{k=1}^{n} H_{2, k}(t)\right\|^{2}+2\left(\sum_{k=1}^{n} e^{-\delta k}\right)\left(\sum_{k=1}^{n} e^{\delta k} E\left\|H_{2, k}(t)\right\|^{2}\right) \\
& \leq C_{2}(M, \delta)\left\|h_{2}\right\|_{S^{2}}^{2},
\end{aligned}
$$

where $C_{2}(M, \delta)$ depends only on the fixed constants $M$ and $\delta$.

Let us show that each $H_{2, k} \in P A P_{0}\left(L^{2}(\mathbb{P}, \mathbb{H})\right)$. For that, note that the above inequality

$$
\begin{aligned}
E\left\|H_{2, k}(t)\right\|^{2} & \leq M^{2}\left(\int_{k-1}^{k} e^{-2 \delta \tau} d \tau\right)\left(\int_{t-k}^{t-k+1} E\left\|h_{2}(\tau)\right\|^{2} d \tau\right) \\
& \leq C_{2}(M, \delta)\left(\int_{t-k}^{t-k+1} E\left\|h_{2}(\tau)\right\|^{2} d \tau\right)
\end{aligned}
$$


and hence $H_{2, k} \in P A P_{0}\left(L^{2}(\mathbb{P}, \mathbb{H})\right)$, as $h_{2}^{b} \in P A P_{0}\left(L^{2}\left(0,1 ; L^{2}(\mathbb{P}, \mathbb{H})\right)\right)$.

Thus we conclude that each $H_{k}(t) \in P A P\left(L^{2}(\mathbb{P}, \mathbb{H})\right)$ and hence $\sum_{k=1}^{\infty} H_{k}(t) \in$ $P A P\left(L^{2}(\mathbb{P}, \mathbb{H})\right)$. Consequently its uniform limit

$$
H(t)=\sum_{k=1}^{\infty} H_{k}(t) \in P A P\left(L^{2}(\mathbb{P}, \mathbb{H})\right)
$$

by Lemma 2.8. The proof is complete.

Lemma 3.2. If $f \in P A P S^{2}\left(L^{2}(\mathbb{P}, \mathbb{H})\right) \cap C\left(\mathbb{R}, L^{2}(\mathbb{P}, \mathbb{H})\right.$ and if $F$ is the function defined by

$$
F(t):=\int_{-\infty}^{t} T(t-\tau) f(\tau) d W(\tau)
$$

for each $t \in \mathbb{R}$, then $F \in P A P\left(L^{2}(\mathbb{P}, \mathbb{H})\right)$.

Proof. Since $f \in P A P S^{2}\left(L^{2}(\mathbb{P}, \mathbb{H})\right) \cap C\left(\mathbb{R}, L^{2}(\mathbb{P}, \mathbb{H})\right.$, write

$$
f=f_{1}+f_{2},
$$

where

$$
f_{1}^{b} \in A P\left(L^{2}\left(0,1 ; L^{2}(\mathbb{P}, \mathbb{H})\right)\right) \cap C\left(\mathbb{R}, L^{2}\left(0,1 ; L^{2}(\mathbb{P}, \mathbb{H})\right)\right)
$$

and

$$
f_{2}^{b} \in P A P_{0}\left(L^{2}\left(0,1 ; L^{2}(\mathbb{P}, \mathbb{H})\right)\right) \cap C\left(\mathbb{R}, L^{2}\left(0,1 ; L^{2}(\mathbb{P}, \mathbb{H})\right)\right),
$$

then $F(t)$ can be decomposed as

$$
F(t)=\int_{-\infty}^{t} T(t-\tau) f_{1}(\tau) d W(\tau)+\int_{-\infty}^{t} T(t-\tau) f_{2}(\tau) d W(\tau) .
$$

Define for all $k=1,2,3, \ldots$, the sequence of integral operators

$$
F_{k}(t):=\int_{k-1}^{k} T(\tau) f_{1}(t-\tau) d W(\tau)+\int_{k-1}^{k} T(\tau) f_{2}(t-\tau) d W(\tau) .
$$

Set

$$
F_{1, k}(t)=\int_{k-1}^{k} T(\tau) f_{1}(t-\tau) d W(\tau)=\int_{t-k}^{t-k+1} T(t-\tau) f_{1}(\tau) d W(\tau)
$$

and

$$
F_{2, k}(t)=\int_{k-1}^{k} T(\tau) f_{2}(t-\tau) d W(\tau)=\int_{t-k}^{t-k+1} T(t-\tau) f_{2}(\tau) d W(\tau)
$$

for each $t \in \mathbb{R}$ and $k=1,2,3, \ldots$ Next we show that $F_{1, k} \in A P\left(L^{2}(\mathbb{P}, \mathbb{H})\right)$ and $F_{2, k} \in P A P_{0}\left(L^{2}(\mathbb{P}, \mathbb{H})\right)$. 
To prove that $F_{1, k} \in A P\left(L^{2}(\mathbb{P}, \mathbb{H})\right)$. Using an estimate on the Ito integral established in [28], it follows that

$$
\begin{aligned}
E\left\|F_{1, k}(t)\right\|^{2} & \leq E\left\|\int_{t-k}^{t-k+1} T(t-\tau) f_{1}(\tau) d W(\tau)\right\|^{2} \\
& \leq \int_{t-k}^{t-k+1}\|T(t-\tau)\|^{2} E\left\|f_{1}(\tau)\right\|^{2} d \tau \\
& \leq M^{2} \int_{t-k}^{t-k+1} e^{-2 \delta(t-\tau)} E\left\|f_{1}(\tau)\right\|^{2} d \tau \\
& \leq M^{2} \int_{k-1}^{k} e^{-2 \delta \tau} E\left\|f_{1}(t-\tau)\right\|^{2} d \tau \\
& \leq M^{2} \sup _{\tau \in[k-1, k]} e^{-2 \delta \tau} \int_{k-1}^{k} E\left\|f_{1}(t-\tau)\right\|^{2} d \tau \\
& \leq M^{2} e^{-2 \delta k} e^{2 \delta}\left\|f_{1}\right\|_{S^{2}}^{2}
\end{aligned}
$$

Since the series

$$
M^{2} e^{2 \delta}\left\|f_{1}\right\|_{S^{2}}^{2} \sum_{k=1}^{\infty} e^{-2 \delta k}<\infty,
$$

we deduce from the well-known Weirstrass test that the series $\sum_{k=1}^{\infty} F_{1, k}(t)$ is uniformly convergent on $\mathbb{R}$. Now let

$$
F_{1}(t):=\sum_{k=1}^{\infty} F_{1, k}(t), \quad t \in \mathbb{R} .
$$

Observe that

$$
F_{1}(t):=\int_{-\infty}^{t} T(t-\tau) f_{1}(\tau) d W(\tau), \quad t \in \mathbb{R}
$$

and hence $F_{1}(t) \in C\left(\mathbb{R}, L^{2}(\mathbb{P}, \mathbb{H})\right)$. Moreover, for any $t \in \mathbb{R}$, we have

$$
\begin{aligned}
E\left\|F_{1}(t)\right\|^{2} & \leq 2 E\left\|F_{1}(t)-\sum_{k=1}^{n} F_{1, k}(t)\right\|^{2}+2\left(\sum_{k=1}^{n} e^{-\delta k}\right)\left(\sum_{k=1}^{n} e^{\delta k} E\left\|F_{1, k}(t)\right\|^{2}\right) \\
& \leq \widetilde{C}_{1}(M, \delta)\left\|f_{1}\right\|_{S^{2}}^{2}
\end{aligned}
$$

where $\widetilde{C}_{1}(M, \delta)$ depends only on the fixed constants $M$ and $\delta$.

Let us show that each $F_{1, k} \in A P\left(L^{2}(\mathbb{P}, \mathbb{H})\right)$.

Indeed, by $f_{1}^{b} \in A P\left(L^{2}\left(0,1 ; L^{2}(\mathbb{P}, \mathbb{H})\right)\right)$, given $\varepsilon>0$, one can find $l(\varepsilon)>0$ such that any interval of length $l(\varepsilon)$ contains at least $s^{\prime}$ with the property that

$$
\int_{t}^{t+1} E\left\|f_{1}\left(s+s^{\prime}\right)-f_{1}(s)\right\|^{2} d s<\varepsilon
$$


for each $t \in \mathbb{R}$. On the other hand, using the inequality (3.4), exponential stable of $T(t)_{t \geq 0}$ and the Ito integral, we obtain that

$$
\begin{aligned}
E & \left\|F_{1, k}\left(t+s^{\prime}\right)-F_{1, k}(t)\right\|^{2} \\
& =E\left\|\int_{k-1}^{k} T(\tau)\left[f_{1}\left(t+s^{\prime}-\tau\right)-f_{1}(t-\tau)\right] d W(\tau)\right\|^{2} \\
& \leq \int_{k-1}^{k}\|T(\tau)\|^{2} E\left\|f_{1}\left(t+s^{\prime}-\tau\right)-f_{1}(t-\tau)\right\|^{2} d \tau \\
& \leq M^{2} \int_{k-1}^{k} e^{-2 \delta \tau} E\left\|f_{1}\left(t+s^{\prime}-\tau\right)-f_{1}(t-\tau)\right\|^{2} d \tau \\
& \leq M^{2} \sup _{\tau \in[k-1, k]} e^{-2 \delta \tau} \int_{k-1}^{k} E\left\|f_{1}\left(t+s^{\prime}-\tau\right)-f_{1}(t-\tau)\right\|^{2} d \tau \\
& \leq M^{2} e^{-2 \delta k} e^{2 \delta} \int_{t-k}^{t-k+1} E\left\|f_{1}\left(s+s^{\prime}\right)-f_{1}(s)\right\|^{2} d s \\
& <M^{2} e^{-2 \delta k} e^{2 \delta} \varepsilon .
\end{aligned}
$$

Therefore, we deduce that $F_{1, k} \in A A\left(L^{2}(\mathbb{P}, \mathbb{H})\right)$.

Next, we will prove that $F_{2, k} \in P A P_{0}\left(L^{2}(\mathbb{P}, \mathbb{H})\right)$. It is obvious that $F_{2, k} \in$ $B C\left(\mathbb{R}, L^{2}(\mathbb{P}, \mathbb{H})\right.$, the left task is to show that

$$
\lim _{r \rightarrow \infty} \frac{1}{2 r} \int_{-r}^{r} E\left\|F_{2, k}(t)\right\|^{2} d t=0
$$

for each $t \in \mathbb{R}$ and $k=1,2,3, \ldots$ Then, by using the exponential stable of $U(t, s)_{t \geq s}$ and the Ito integral, it follows that

$$
\begin{aligned}
E\left\|F_{2, k}(t)\right\|^{2} & =E\left\|\int_{t-k}^{t-k+1} T(t-\tau) f_{2}(\tau) d \tau\right\|^{2} \\
& \leq M^{2} \int_{t-k}^{t-k+1} e^{-2 \delta(t-\tau)} E\left\|f_{2}(\tau)\right\|^{2} d \tau \\
& \leq M^{2} \int_{k-1}^{k} e^{-2 \delta \tau} E\left\|f_{2}(t-\tau)\right\|^{2} d \tau \\
& \leq M^{2} \sup _{\tau \in[k-1, k]} e^{-2 \delta \tau} \int_{k-1}^{k} E\left\|f_{2}(t-\tau)\right\|^{2} d \tau \\
& \leq M^{2} e^{-2 \delta k} e^{2 \delta}\left\|f_{2}\right\|_{S^{2}}^{2} .
\end{aligned}
$$

Since the series

$$
M^{2} e^{2 \delta}\left\|f_{2}\right\|_{S^{2}}^{2} \sum_{k=1}^{\infty} e^{-2 \delta k}<\infty,
$$

we deduce from the well-known Weirstrass test that the series $\sum_{k=1}^{\infty} F_{2, k}(t)$ is uniformly convergent on $\mathbb{R}$. Now let

$$
F_{2}(t):=\sum_{k=1}^{\infty} F_{2, k}(t), \quad t \in \mathbb{R} .
$$




$$
F_{2}(t)=\int_{-\infty}^{t} T(t-\tau) f_{2}(\tau) d W(\tau), \quad t \in \mathbb{R}
$$

and hence $F_{2}(t) \in C\left(\mathbb{R}, L^{2}(\mathbb{P}, \mathbb{H})\right)$. Moreover, for any $t \in \mathbb{R}$, we have

$$
\begin{aligned}
E\left\|F_{2}(t)\right\|^{2} & \leq 2 E\left\|F_{2}(t)-\sum_{k=1}^{n} F_{2, k}(t)\right\|^{2}+2\left(\sum_{k=1}^{n} e^{-\delta k}\right)\left(\sum_{k=1}^{n} e^{\delta k} E\left\|F_{2, k}(t)\right\|^{2}\right) \\
& \leq \widetilde{C}_{2}(M, \delta)\left\|f_{2}\right\|_{S^{2}}^{2}
\end{aligned}
$$

where $\widetilde{C}_{2}(M, \delta)$ depends only on the fixed constants $M$ and $\delta$.

Let us show that each $F_{2, k} \in P A P_{0}\left(L^{2}(\mathbb{P}, \mathbb{H})\right)$. For that, note that the above inequality

$$
\begin{aligned}
E\left\|F_{2, k}(t)\right\|^{2} & \leq 2 M^{2} e^{-2 \delta k} e^{2 \delta} \int_{k-1}^{k} E\left\|f_{2}(t-\tau)\right\|^{2} d \tau \\
& \leq \widetilde{C}_{2}(M, \delta) \int_{k-1}^{k} E\left\|f_{2}(t-\tau)\right\|^{2} d \tau
\end{aligned}
$$

and hence $F_{2, k} \in P A P_{0}\left(L^{2}(\mathbb{P}, \mathbb{H})\right)$, as $f_{2}^{b} \in P A P_{0}\left(L^{2}\left(0,1 ; L^{2}(\mathbb{P}, \mathbb{H})\right)\right)$.

Thus we conclude that each $F_{k}(t) \in P A P\left(L^{2}(\mathbb{P}, \mathbb{H})\right)$ and hence $\sum_{k=1}^{\infty} F_{k}(t) \in$ $P A P\left(L^{2}(\mathbb{P}, \mathbb{H})\right)$. Consequently its uniform limit

$$
F(t)=\sum_{k=1}^{\infty} F_{k}(t) \in P A P\left(L^{2}(\mathbb{P}, \mathbb{H})\right),
$$

by Lemma 2.8. The proof is complete.

Now, we establish the existence and uniqueness theorem of pseudo almost periodic mild solutions to neutral partial stochastic differential equation (1.1). For that, we make the following hypotheses:

(H1) The functions $\left.g \in P A P\left(\mathbb{R} \times L^{2}(\mathbb{P}, \mathbb{H})\right) \cap C\left(\mathbb{R} \times L^{2}(\mathbb{P}, \mathbb{H})\right), L^{2}(\mathbb{P}, \mathbb{H})\right), h, f \in$ $P A P S^{2}\left(\mathbb{R} \times L^{2}(\mathbb{P}, \mathbb{H})\right) \cap C\left(\mathbb{R} \times L^{2}(\mathbb{P}, \mathbb{H}), L^{2}(\mathbb{P}, \mathbb{H})\right)$.

(H2) The functions $g, h, f$ are Lipschitz with respect to the second argument uniformly in the first argument in the sense that: there exist $L_{g}, L_{h}, L_{f}>0$ such that

$$
\begin{aligned}
& E\|g(t, x)-g(t, y)\|^{2} \leq L_{g} E\|x-y\|^{2}, \\
& E\|h(t, x)-h(t, y)\|^{2} \leq L_{h} E\|x-y\|^{2},
\end{aligned}
$$

and

$$
E\|f(t, x)-f(t, y)\|^{2} \leq L_{f} E\|x-y\|^{2}
$$

for all $t \in \mathbb{R}$ and each $x, y \in L^{2}(\mathbb{P}, \mathbb{H})$.

Definition 3.3. An $\mathcal{F}_{t}$-progressively measurable stochastic process $x: \mathbb{R} \rightarrow$ $L^{2}(\mathbb{P}, \mathbb{H})$ is called a mild solution of the system $(1.1)$ if $x(t)$ satisfies

$$
\begin{aligned}
x(t)- & g(t, x(t))=T(t-s)[x(s)-g(s, x(s))] \\
& +\int_{s}^{t} T(t-\tau) h(\tau, x(\tau)) d \tau+\int_{s}^{t} T(t-\tau) f(\tau, x(\tau)) d W(\tau)
\end{aligned}
$$

for all $t \geq s$ and all $s \in \mathbb{R}$. 
Theorem 3.4. Assume that (H1)-(H2) hold. If

$$
3\left[L_{g}+\frac{M^{2}}{\delta^{2}} L_{h}+\frac{M^{2}}{2 \delta} L_{f}\right]<1 .
$$

then Eq. (1.1) admits a unique pseudo almost periodic mild solution on $\mathbb{R}$.

Proof. Consider the nonlinear operator $\Lambda$ on $B C\left(\mathbb{R}, L^{2}(\mathbb{P}, \mathbb{H})\right)$ defined by

$$
\begin{aligned}
(\Lambda x)(t)= & g(t, x(t))+\int_{-\infty}^{t} T(t-\tau) h(\tau, x(\tau) d \tau \\
& +\int_{-\infty}^{t} T(t-\tau) f(\tau, x(\tau) d W(\tau), \quad t \in \mathbb{R} .
\end{aligned}
$$

Let $x(\cdot) \in P A P\left(L^{2}(\mathbb{P}, \mathbb{H})\right)$, then $g(\cdot, x(\cdot)) \in P A P\left(L^{2}(\mathbb{P}, \mathbb{H})\right)$, by Lemma 2.10, thanks to assumptions (H1)-(H2). Together with Lemma 2.18, we deduce that $h(\cdot, x(\cdot)), f(\cdot, x(\cdot)) \in P A P S^{2}\left(L^{2}(\mathbb{P}, \mathbb{H})\right)$. It is easy to check that $h(\cdot, x(\cdot)), f(\cdot, x(\cdot))$ is in $C\left(\mathbb{R}, L^{2}(\mathbb{P}, \mathbb{H})\right)$. Applying Lemma 3.1,Lemma 3.2 for $h(\cdot)=h(\cdot, x(\cdot)), f(\cdot)=$ $f(\cdot, x(\cdot))$, it follows that the operator $\Lambda$ is well defined and maps in $P A P\left(L^{2}(\mathbb{P}, \mathbb{H})\right)$ into itself.

Let $x, y \in P A P\left(L^{2}(\mathbb{P}, \mathbb{H})\right)$, then $(\mathrm{H} 1)$ - $(\mathrm{H} 2)$ yield that

$$
\begin{aligned}
E\|(\Lambda x)(t)-(\Lambda y)(t)\|^{2} \leq & 3 E\|g(t, x(t))-g(t, y(t))\|^{2} \\
& +3 E\left\|\int_{-\infty}^{t} T(t-s)[h(s, x(s))-h(s, y(s))] d s\right\|^{2} \\
& +3 E\left\|\int_{-\infty}^{t} T(t-s)[f(s, x(s))-f(s, y(s))] d W(s)\right\|^{2} .
\end{aligned}
$$

By using the Cauchy-Schwarz inequality, we first evaluate the first second term of the right-hand side

$$
\begin{aligned}
3 E\|g(t, x(t))-g(t, y(t))\|^{2} & \leq 3 L_{g} E\|x(t)-y(t)\|^{2} \\
& \leq 3 L_{q}\|x-y\|_{\infty}^{2}
\end{aligned}
$$

and

$$
\begin{aligned}
3 E & \left\|\int_{-\infty}^{t} T(t-s)[h(s, x(s))-h(s, y(s))] d s\right\|^{2} \\
& \leq 3 E\left(\int_{-\infty}^{t}\|T(t-s)\|\|h(s, x(s))-h(s, y(s))\| d s\right)^{2} \\
& \leq 3 M^{2} E\left(\int_{-\infty}^{t} e^{-\delta(t-s)}\|h(s, x(s))-h(s, y(s))\| d s\right)^{2} \\
& \leq 3 M^{2}\left(\int_{-\infty}^{t} e^{-\delta(t-s)} d s\right)\left(\int_{0}^{t} e^{-\delta(t-s)} E\|h(s, x(s))-h(s, y(s))\|^{2} d s\right)
\end{aligned}
$$




$$
\begin{aligned}
& \leq 3 M_{\alpha}^{2} L_{h}\left(\int_{-\infty}^{t} e^{-\delta(t-s)} d s\right)\left(\int_{-\infty}^{t} e^{-\delta(t-s)} E\|x(s)-y(s)\|^{2} d s\right) \\
& \leq 3 M^{2} L_{h}\left(\int_{-\infty}^{t} e^{-\delta(t-s)} d s\right)^{2} \sup _{s \in \mathbb{R}} E\|x(s)-y(s)\|^{2} \\
& \leq \frac{3 M^{2}}{\delta^{2}} L_{h}\|x-y\|_{\infty}^{2} .
\end{aligned}
$$

As to the last term, by the Ito integral, we get

$$
\begin{aligned}
3 E & \left\|\int_{-\infty}^{t} T(t-s)[f(s, x(s))-f(s, y(s))] d W(s)\right\|^{2} \\
& \leq 3 \int_{-\infty}^{t}\|T(t-s)\|^{2} E\|f(s, x(s))-f(s, y(s))\|^{2} d s \\
& \leq 3 M^{2} \int_{-\infty}^{t} e^{-2 \delta(t-s)} E\|f(s, x(s))-f(s, y(s))\|^{2} d s \\
& \leq 3 M^{2} L_{f} \int_{-\infty}^{t} e^{-2 \delta(t-s)} E\|x(s)-y(s)\|^{2} d s \\
& \leq 3 M^{2} L_{f} \int_{-\infty}^{t} e^{-2 \delta(t-s)} d s \sup _{s \in \mathbb{R}} E\|x(s)-y(s)\|^{2} \\
& \leq \frac{3 M^{2}}{2 \delta} L_{f}\|x-y\|_{\infty}^{2} .
\end{aligned}
$$

Thus, by combining the above inequality together, we obtain that, for each $t \in \mathbb{R}$,

$$
E\|(\Lambda x)(t)-(\Lambda y)(t)\|^{2} \leq 3\left[L_{g}+\frac{M^{2}}{\delta^{2}} L_{h}+\frac{M^{2}}{2 \delta} L_{f}\right]\|x-y\|_{\infty}^{2} .
$$

Hence

$$
\|\Lambda x-\Lambda y\|_{\infty} \leq \sqrt{L_{0}}\|x-y\|_{\infty},
$$

where $L_{0}=3\left[L_{g}+\frac{M^{2}}{\delta^{2}} L_{h}+\frac{M^{2}}{2 \delta} L_{f}\right]<1$, then the operator $\Lambda$ becomes a strict contraction. By the Banach contraction principle, we draw a conclusion that there exists a unique fixed point $x(\cdot)$ for $\Lambda$ in $P A P\left(L^{2}(\mathbb{P}, \mathbb{H})\right)$.

Finally, to prove that $x$ satisfies (3.5) for all $t \geq s$, all $s \in \mathbb{R}$. For this, we let

$$
\begin{aligned}
x(s)= & g(s, x(s))+\int_{-\infty}^{s} T(t-\tau) h(\tau, x(\tau)) d \tau \\
& +\int_{-\infty}^{s} T(t-\tau) f(\tau, x(\tau)) d W(\tau), \quad s \in \mathbb{R} .
\end{aligned}
$$

Multiply both sides of (3.7) by $T(s)$ for all $s \in \mathbb{R}$, then

$$
\begin{aligned}
T(s) x(s)= & T(s) g(s, x(s))+\int_{-\infty}^{s} T(t-\tau) h(\tau, x(\tau)) d \tau \\
& +\int_{-\infty}^{s} T(t-\tau) f(\tau, x(\tau)) d W(\tau)
\end{aligned}
$$




$$
\begin{aligned}
= & T(s) g(s, x(s))+\int_{-\infty}^{t} T(t-\tau) h(\tau, x(\tau)) d \tau \\
& -\int_{s}^{t} T(t-\tau) h(\tau, x(\tau)) d \tau \\
& +\int_{-\infty}^{t} T(t-\tau) f(\tau, x(\tau)) d W(\tau)-\int_{s}^{t} T(t-\tau) f(\tau, x(\tau)) d W(\tau) \\
= & T(s) g(s, x(s))+x(t)-g(t, x(t)) \\
& -\int_{s}^{t} T(t-\tau) h(\tau, x(\tau)) d \tau-\int_{s}^{t} T(t-\tau) f(\tau, x(\tau)) d W(\tau) .
\end{aligned}
$$

Hence $x \in P A P\left(L^{2}(\mathbb{P}, \mathbb{H})\right)$ is a unique mild solution to Eq. (1.1). The proof is complete.

Next, we establish the existence and uniqueness theorem of pseudo almost periodic mild solutions to perturb neutral partial stochastic differential equation (1.2). For that, we make the following hypotheses:

$(\mathrm{P} 1) L^{2}(\mathbb{P}, \mathbb{K}) \hookrightarrow L^{2}(\mathbb{P}, \mathbb{H})$ is continuously embedded, where $\mathbb{K}:=[D(A)]$ (equipped with graph norm).

(P2) The functions $g \in P A P\left(\mathbb{R} \times L^{2}(\mathbb{P}, \mathbb{K})\right) \cap C\left(\mathbb{R} \times L^{2}(\mathbb{P}, \mathbb{H}), L^{2}(\mathbb{P}, \mathbb{K})\right), h, f \in$ $\left.P A P S^{2}\left(\mathbb{R} \times L^{2}(\mathbb{P}, \mathbb{K})\right) \cap C\left(\mathbb{R} \times L^{2}(\mathbb{P}, \mathbb{H})\right), L^{2}(\mathbb{P}, \mathbb{K})\right)$.

(P3) The functions $g, h, f$ are Lipschitz with respect to the second argument uniformly in the first argument in the sense that: there exist $\tilde{L}_{g}, \tilde{L}_{h}, \tilde{L}_{f}>0$ such that

$$
\begin{aligned}
& E\|g(t, x)-g(t, y)\|_{\mathbb{K}}^{2} \leq \tilde{L}_{g} E\|x-y\|^{2}, \\
& E\|h(t, x)-h(t, y)\|_{\mathbb{K}}^{2} \leq \tilde{L}_{h} E\|x-y\|^{2},
\end{aligned}
$$

and

$$
E\|f(t, x)-f(t, y)\|_{\mathbb{K}}^{2} \leq \tilde{L}_{f} E\|x-y\|^{2}
$$

for all $t \in \mathbb{R}$ and each $x, y \in L^{2}(\mathbb{P}, \mathbb{H})$.

(P4) $B_{i} \in L\left(L^{2}(\mathbb{P}, \mathbb{K}), L^{2}(\mathbb{P}, \mathbb{H})\right)$ with $\max _{i=1,2,3}\left\{\left\|B_{i}\right\|_{L\left(L^{2}(\mathbb{P}, \mathbb{K}), L^{2}(\mathbb{P}, \mathbb{H})\right)}\right\}=\mu$.

Definition 3.5. An $\mathcal{F}_{t}$-progressively measurable stochastic process $x: \mathbb{R} \rightarrow$ $L^{2}(\mathbb{P}, \mathbb{K})$ is called a mild solution of the system (3.1) if $x(t)$ satisfies

$$
\begin{aligned}
x(t)- & g\left(t, B_{1} x(t)\right)=T(t-s)\left[x(s)-g\left(s, B_{1} x(s)\right)\right] \\
& +\int_{s}^{t} T(t-\tau) h\left(\tau, B_{2} x(\tau)\right) d \tau+\int_{s}^{t} T(t-\tau) f\left(\tau, B_{3} x(\tau)\right) d W(\tau)
\end{aligned}
$$

for all $t \geq s$ and all $s \in \mathbb{R}$.

Theorem 3.6. Assume that (P1)-(P4) hold. If

$$
3\left[\tilde{L}_{g}+\frac{M^{2}}{\delta^{2}} \tilde{L}_{h}+\frac{M^{2}}{2 \delta} \tilde{L}_{f}\right] \mu^{2}<1 .
$$

then Eq. (1.2) admits a unique pseudo almost periodic mild solution on $\mathbb{R}$. 
Proof. Consider the nonlinear operator $\widetilde{\Lambda}$ on $B C\left(\mathbb{R}, L^{2}(\mathbb{P}, \mathbb{K})\right)$ defined by

$$
\begin{aligned}
(\widetilde{\Lambda} x)(t)= & g\left(t, B_{1} x(t)\right)+\int_{-\infty}^{t} T(t-\tau) h\left(\tau, B_{2} x(\tau)\right) d \tau \\
& +\int_{-\infty}^{t} T(t-\tau) f\left(\tau, B_{3} x(\tau)\right) d W(\tau), \quad t \in \mathbb{R}
\end{aligned}
$$

Let $x(\cdot) \in P A P\left(L^{2}(\mathbb{P}, \mathbb{K})\right)$, then $g\left(\cdot, B_{1} x(\cdot)\right) \in P A P\left(L^{2}(\mathbb{P}, \mathbb{K})\right)$, Lemma 2.10, Lemma 2.11, thanks to assumptions (P1)-(P4). Together with Lemma 2.18, Lemma 2.19, we deduce that $h\left(\cdot, B_{2} x(\cdot)\right), f\left(\cdot, B_{3} x(\cdot)\right) \in P A P S^{2}\left(L^{2}(\mathbb{P}, \mathbb{H})\right)$. It is easy to check that $h\left(\cdot, B_{2} x(\cdot)\right), f\left(\cdot, B_{3} x(\cdot)\right) \in C\left(\mathbb{R}, L^{2}(\mathbb{P}, \mathbb{H})\right)$. Applying Lemma 3.1, Lemma 3.2 for $h(\cdot)=h\left(\cdot, B_{2} x(\cdot)\right), f(\cdot)=f\left(\cdot, B_{3} x(\cdot)\right)$, it follows that the operator $\widetilde{\Lambda}$ is well defined and maps in $\operatorname{PAP}\left(L^{2}(\mathbb{P}, \mathbb{K})\right)$ into itself.

Let $x, y \in P A P\left(L^{2}(\mathbb{P}, \mathbb{K})\right)$, then $(\mathrm{P} 2)$-(P4) yield that

$$
\begin{aligned}
E \| & (\widetilde{\Lambda} x)(t)-(\widetilde{\Lambda} y)(t) \|_{\mathbb{K}}^{2} \\
\leq & 3 E\left\|g\left(t, B_{1} x(t)\right)-g\left(t, B_{1} y(t)\right)\right\|_{\mathbb{K}}^{2} \\
& +3 E\left\|\int_{-\infty}^{t} T(t-s)\left[h\left(s, B_{2} x(s)\right)-h\left(s, B_{2} y(s)\right)\right] d s\right\|_{\mathbb{K}}^{2} \\
& +3 E\left\|\int_{-\infty}^{t} T(t-s)\left[f\left(s, B_{3} x(s)\right)-f\left(s, B_{3} y(s)\right)\right] d W(s)\right\|_{\mathbb{K}}^{2} .
\end{aligned}
$$

By using the Cauchy-Schwarz inequality, we first evaluate the first second term of the right-hand side

$$
\begin{aligned}
3 E\left\|g\left(t, B_{1} x(t)\right)-g\left(t, B_{1} y(t)\right)\right\|_{\mathbb{K}}^{2} & \leq 3 \tilde{L}_{g} \mu^{2} E\|x(t)-y(t)\|_{\mathbb{K}}^{2} \\
& \leq 3 \tilde{L}_{q} \mu^{2}\|x-y\|_{\infty, \mathbb{K}}^{2}
\end{aligned}
$$

and

$$
\begin{aligned}
3 E \| & \int_{-\infty}^{t} T(t-s)\left[h\left(s, B_{2} x(s)\right)-h\left(s, B_{2} y(s)\right)\right] d s \|_{\mathbb{K}}^{2} \\
\leq & 3 E\left(\int_{-\infty}^{t}\|T(t-s)\|\left\|h\left(s, B_{2} x(s)\right)-h\left(s, B_{2} y(s)\right)\right\|_{\mathbb{K}} d s\right)^{2} \\
\leq & 3 M^{2} E\left(\int_{-\infty}^{t} e^{-\delta(t-s)}\left\|h\left(s, B_{2} x(s)\right)-h\left(s, B_{2} y(s)\right)\right\|_{\mathbb{K}} d s\right)^{2} \\
\leq & 3 M^{2}\left(\int_{-\infty}^{t} e^{-\delta(t-s)} d s\right) \\
& \times\left(\int_{0}^{t} e^{-\delta(t-s)} E\left\|h\left(s, B_{2} x(s)\right)-h\left(s, B_{2} y(s)\right)\right\|_{\mathbb{K}}^{2} d s\right) \\
\leq & 3 M^{2} \tilde{L}_{h} \mu^{2}\left(\int_{-\infty}^{t} e^{-\delta(t-s)} d s\right)\left(\int_{-\infty}^{t} e^{-\delta(t-s)} E\|x(s)-y(s)\|_{\mathbb{K}}^{2} d s\right)
\end{aligned}
$$




$$
\begin{aligned}
& \leq 3 M^{2} \tilde{L}_{h} \mu^{2}\left(\int_{-\infty}^{t} e^{-\delta(t-s)} d s\right)^{2} \sup _{s \in \mathbb{R}} E\|x(s)-y(s)\|_{\mathbb{K}}^{2} \\
& \leq \frac{3 M^{2}}{\delta^{2}} \tilde{L}_{h} \mu^{2}\|x-y\|_{\infty, \mathbb{K}}^{2} .
\end{aligned}
$$

As to the last term, by the Ito integral, we get

$$
\begin{aligned}
3 E & \left\|\int_{-\infty}^{t} T(t-s)\left[f\left(s, B_{3} x(s)\right)-f\left(s, B_{3} y(s)\right)\right] d W(s)\right\|_{\mathbb{K}}^{2} \\
& \leq 3 \int_{-\infty}^{t}\|T(t-s)\|^{2} E\left\|f\left(s, B_{3} x(s)\right)-f\left(s, B_{3} y(s)\right)\right\|_{\mathbb{K}}^{2} d s \\
& \leq 3 M^{2} \int_{-\infty}^{t} e^{-2 \delta(t-s)} E\left\|f\left(s, B_{3} x(s)\right)-f\left(s, B_{3} y(s)\right)\right\|_{\mathbb{K}}^{2} d s \\
& \leq 3 M^{2} \tilde{L}_{f} \mu^{2} \int_{-\infty}^{t} e^{-2 \delta(t-s)} E\|x(s)-y(s)\|_{\mathbb{K}}^{2} d s \\
& \leq 3 M^{2} \tilde{L}_{f} \mu^{2} \int_{-\infty}^{t} e^{-2 \delta(t-s)} d s \sup _{s \in \mathbb{R}} E\|x(s)-y(s)\|_{\mathbb{K}}^{2} \\
& \leq \frac{3 M^{2}}{2 \delta} \tilde{L}_{f} \mu^{2}\|x-y\|_{\infty, \mathbb{K}}^{2} \cdot
\end{aligned}
$$

Thus, by combining the above inequality together, we obtain that, for each $t \in \mathbb{R}$,

$$
\begin{aligned}
& E\|(\Psi x)(t)-(\Psi y)(t)\|_{\mathbb{K}}^{2} \\
& \quad \leq 3\left[\tilde{L}_{g}+\frac{M^{2}}{\delta^{2}} \tilde{L}_{h}+\frac{M^{2}}{2 \delta} \tilde{L}_{f}\right] \mu^{2}\|x-y\|_{\infty, \mathbb{K}}^{2} .
\end{aligned}
$$

Hence

$$
\|\widetilde{\Lambda} x-\widetilde{\Lambda} y\|_{\infty, \mathbb{K}} \leq \sqrt{L_{0}^{*}}\|x-y\|_{\infty, \mathbb{K}}
$$

where $L_{0}^{*}=3\left[\tilde{L}_{g}+\frac{M^{2}}{\delta^{2}} \tilde{L}_{h}+\frac{M^{2}}{2 \delta} \tilde{L}_{f}\right] \mu^{2}<1$, then the operator $\widetilde{\Lambda}$ becomes a strict contraction. By the Banach contraction principle, we draw a conclusion that there exists a unique fixed point $x(\cdot)$ for $\widetilde{\Lambda}$ in $P A P\left(L^{2}(\mathbb{P}, \mathbb{K})\right)$.

Finally, to prove that $x$ satisfies (3.8) for all $t \geq s$, all $s \in \mathbb{R}$. For this, we let

$$
\begin{aligned}
x(s)= & g\left(s, B_{1} x(s)\right)+\int_{-\infty}^{s} T(t-\tau) h\left(\tau, B_{2} x(\tau)\right) d \tau \\
& +\int_{-\infty}^{s} T(t-\tau) f\left(\tau, B_{3} x(\tau)\right) d W(\tau), \quad s \in \mathbb{R} .
\end{aligned}
$$

Multiply both sides of (3.10) by $T(s)$ for all $s \in \mathbb{R}$, then

$$
\begin{aligned}
T(s) x(s)= & T(s) g\left(s, B_{1} x(s)\right)+\int_{-\infty}^{s} T(t-\tau) h\left(\tau, B_{2} x(\tau)\right) d \tau \\
& +\int_{-\infty}^{s} T(t-\tau) f\left(\tau, B_{3} x(\tau)\right) d W(\tau) \\
= & T(s) g\left(s, B_{1} x(s)\right)+\int_{-\infty}^{t} T(t-\tau) h\left(\tau, B_{2} x(\tau)\right) d \tau
\end{aligned}
$$




$$
\begin{aligned}
& -\int_{s}^{t} T(t-\tau) h\left(\tau, B_{2} x(\tau)\right) d \tau \\
& +\int_{-\infty}^{t} T(t-\tau) f\left(\tau, B_{3} x(\tau)\right) d W(\tau)-\int_{s}^{t} T(t-\tau) f\left(\tau, B_{3} x(\tau)\right) d W(\tau) \\
= & T(s) g\left(s, B_{1} x(s)\right)+x(t)-g\left(t, B_{1} x(t)\right) \\
& -\int_{s}^{t} T(t-\tau) h\left(\tau, B_{2} x(\tau)\right) d \tau-\int_{s}^{t} T(t-\tau) f\left(\tau, B_{3} x(\tau)\right) d W(\tau) .
\end{aligned}
$$

Hence $x \in P A P\left(L^{2}(\mathbb{P}, \mathbb{K})\right)$ is a unique mild solution to Eq. (1.2). The proof is complete.

\section{Application}

Example 4.1. Consider pseudo almost periodic mild solutions to following neutral partial stochastic differential equations of the form

$$
\begin{gathered}
d z(t, x)=\frac{\partial^{2}}{\partial x^{2}} z(t, x) d t+d a_{1}(t, z(t, x)) d t \\
+a_{2}(t, z(t, x)) d t+a_{3}(t, z(t, x)) d W(t), \\
\left.a_{1}(t, z(t, x))\right|_{x=0}=\left.a_{1}(t, z(t, x))\right|_{x=\pi}=0, \quad t \in \mathbb{R}, \\
z(t, 0)=z(t, \pi)=0, \quad t \in \mathbb{R}, x \in[0, \pi],
\end{gathered}
$$

where $W(t)$ is a two-sided standard one-dimensional Brownian motion defined on the filtered probability space $\left(\Omega, \mathcal{F}, \mathbb{P}, \mathcal{F}_{t}\right)$. In this system, $a_{1}$ is pseudo almost periodic continuous function and $a_{i}, i=2,3$, are Stepanov-like pseudo almost periodic continuous functions.

Let $\mathbb{H}=L^{2}([0, \pi])$ with the norm $\|\cdot\|$ and define the operators $A: A(D) \subset$ $\mathbb{H} \rightarrow \mathbb{H}$ by $A v=v^{\prime \prime}$ with the domain $D(A):=\left\{v \in \mathbb{H}: v^{\prime \prime} \in \mathbb{H}, v(0)=v(\pi)=0\right\}$ It is well known that $A$ is the infinitesimal generator of an analytic semigroup $T(t)$ on $\mathbb{H}$ with $(M=\delta=1)$

$$
\|T(t)\| \leq e^{-t} \text { for } t \geq 0 .
$$

Furthermore, $A$ has a discrete spectrum with eigenvalues of the form $-n^{2}, n \in \mathbb{N}$ and normalized eigenfunctions given by $v_{n}(\xi):=\left(\frac{2}{\pi}\right)^{\frac{1}{2}} \sin (n \xi)$. In addition, the following properties hold:

(a) The set of functions $\left\{v_{n}: n \in \mathbb{N}\right\}$ is an orthonormal basis for $\mathbb{H}$;

(b) For $v \in \mathbb{H}, T(t) v=\sum_{n=1}^{\infty} \exp \left(-n^{2} t\right)\left\langle v, v_{n}\right\rangle v_{n}$, and $A v=\sum_{n=1}^{\infty} n^{2}\left\langle v, v_{n}\right\rangle v_{n}$, $v \in D(A)$;

Let $g \in P A P\left(\mathbb{R} \times L^{2}(\mathbb{P}, \mathbb{H})\right) \cap C\left(\mathbb{R} \times L^{2}(\mathbb{P}, \mathbb{H}), L^{2}(\mathbb{P}, \mathbb{H})\right), h, f \in P A P S^{2}(\mathbb{R} \times$ $\left.L^{2}(\mathbb{P}, \mathbb{H})\right) \cap C\left(\mathbb{R} \times L^{2}(\mathbb{P}, \mathbb{H}), L^{2}(\mathbb{P}, \mathbb{H})\right)$, and be defined for $x \in[0, \pi]$ and $t \in \mathbb{R}$ by

$$
\begin{aligned}
& g(t, u)(x)=a_{1}(t, u(t)(x)), \\
& h(t, u)(x)=a_{2}(t, u(t)(x)), \\
& f(t, u)(x)=a_{3}(t, u(t)(x)) .
\end{aligned}
$$


Then, the above equation can be written in the abstract form as the system (1.1). Assume that there exist constants $L_{i}>0, i=1,2,3$ such that

$$
\begin{aligned}
& E\left\|a_{1}(t, u)-a_{1}(t, v)\right\|^{2} \leq L_{1} E\|u-v\|^{2}, \\
& E\left\|a_{2}(t, u)-a_{2}(t, v)\right\|^{2} \leq L_{2} E\|u-v\|^{2}, \\
& E\left\|a_{3}(t, u)-a_{3}(t, v)\right\|^{2} \leq L_{3} E\|u-v\|^{2},
\end{aligned}
$$

for all $t \in \mathbb{R}$ and each $u, v \in L^{2}(\mathbb{P}, \mathbb{H})$.

Consequently all assumptions (H1)-(H2) are satisfied, then by Theorem 3.4, we deduce the following result.

Proposition 4.1. Under the above assumption, if

$$
3\left(L_{1}+L_{2}+L_{3}\right)<1 .
$$

Then, Eq. (4.1)-(4.3) has a unique pseudo almost periodic solution on $\mathbb{R}$.

Example 4.2. Consider pseudo almost periodic mild solutions to following perturbed neutral partial stochastic differential equations of the form

$$
\begin{aligned}
& d z(t, x)=\frac{\partial^{2}}{\partial x^{2}} z(t, x) d t+d a_{1}\left(t, \frac{\partial^{2}}{\partial x^{2}} z(t, x)\right) \\
&+a_{2}\left(t, \frac{\partial^{2}}{\partial x^{2}} z(t, x)\right) d t+a_{3}\left(t, z\left(t, \frac{\partial^{2}}{\partial x^{2}} x\right)\right) d W(t), \\
&\left.a_{1}\left(t, \frac{\partial^{2}}{\partial x^{2}} z(t, x)\right)\right|_{x=0}=\left.a_{1}\left(t, \frac{\partial^{2}}{\partial x^{2}} z(t, x)\right)\right|_{x=\pi}=0, \quad t \in \mathbb{R}, \\
& z(t, 0)=z(t, \pi)=0, \quad t \in \mathbb{R}, x \in[0, \pi],
\end{aligned}
$$

where $\mathbb{H}, A, W(t), a_{i}, i=1,2,3$, be as in Example 4.1. In addition, let $\mathbb{K}:=$ $[D(A)]$ denote the space $D(A)$ endowed with the graph norm $\|\cdot\|=\|\cdot\|+\|A \cdot\|$, then $L^{2}(\mathbb{P}, \mathbb{K}) \hookrightarrow L^{2}(\mathbb{P}, \mathbb{H})$ is continuously embedded.

Define the operators $B_{i}, i=1,2,3$, by $B_{i}=A$ with $D\left(B_{i}\right)=D(A)$, then $B_{i}: L^{2}(\mathbb{P}, \mathbb{K}) \rightarrow L^{2}(\mathbb{P}, \mathbb{H})$ are bounded and $\left\|B_{i}\right\|_{L\left(L^{2}(\mathbb{P}, \mathbb{K}), L^{2}(\mathbb{P}, \mathbb{H})\right)}=1$.

Let $g \in P A P\left(\mathbb{R} \times L^{2}(\mathbb{P}, \mathbb{H})\right) \cap C\left(\mathbb{R} \times L^{2}(\mathbb{P}, \mathbb{H}), L^{2}(\mathbb{P}, \mathbb{K})\right), h, f \in P A P S^{2}(\mathbb{R} \times$ $\left.L^{2}(\mathbb{P}, \mathbb{H})\right) \cap C\left(\mathbb{R} \times L^{2}(\mathbb{P}, \mathbb{H}), L^{2}(\mathbb{P}, \mathbb{K})\right)$, and be defined for $x \in[0, \pi]$ and $t \in \mathbb{R}$ by

$$
\begin{aligned}
& g(t, u)(x)=a_{1}(t, u(t)(x)), \\
& h(t, u)(x)=a_{2}(t, u(t)(x)), \\
& f(t, u)(x)=a_{3}(t, u(t)(x)) .
\end{aligned}
$$

Then, the above equation can be written in the abstract form as the system (1.2). Assume that there exist constants $\tilde{L}_{i}>0, i=1,2,3$ such that

$$
\begin{gathered}
E\left\|a_{1}(t, u)-a_{1}(t, v)\right\|_{\mathbb{K}}^{2} \leq \tilde{L}_{1} E\|u-v\|^{2}, \\
E\left\|a_{2}(t, u)-a_{2}(t, v)\right\|_{\mathbb{K}}^{2} \leq \tilde{L}_{2} E\|u-v\|^{2}, \\
E\left\|a_{3}(t, u)-a_{3}(t, v)\right\|_{\mathbb{K}}^{2} \leq \tilde{L}_{3} E\|u-v\|^{2}
\end{gathered}
$$

for all $t \in \mathbb{R}$ and each $u, v \in L^{2}(\mathbb{P}, \mathbb{H})$.

Consequently all assumptions (P1)-(P4) are satisfied, then by Theorem 3.6, we deduce the following result. 
Proposition 4.3. Under the above assumption, if

$$
3\left(\tilde{L}_{1}+\tilde{L}_{2}+\tilde{L}_{3}\right)<1 .
$$

Then, Eq. (4.4)-(4.6) has a unique pseudo almost periodic solution on $\mathbb{R}$.

Acknowledgement.The authors would like to thank the editor and the reviewers for their constructive comments and suggestions to improve the quality of the paper.

\section{REFERENCES}

1. S. Abbas and D. Bahuguna, Almost periodic solutions of neutral functional differential equations, Comput. Math. Appl. 55 (2008), no. 11, 2593-2601.

2. M. Adimy and K. Ezzinbi, A class of linear partial neutral functional-differential equations with nondense domain, J. Differential Equations 147 (1998), no. 2, 285-332.

3. E. Ait Dads, K. Ezzinbi and O. Arino, Pseudo almost periodic solution for some differential equation in a Banach space, Nonlinear Anal. 28 (1997), no. 7, 1141-1155.

4. L. Arnold and C. Tudor, Stationary and almost periodic solutions of almost periodic affine stochastic differential equations, Stoch. Stoch. Rep. 64 (1998), no. 3-4, 177-193.

5. P.H. Bezandry and T. Diagana, Existence of almost periodic solutions to some stochastic differential equations, Appl. Anal. 86 (2007), no. 7, 819-827.

6. P.H. Bezandry and T. Diagana, Square-mean almost periodic solutions nonautonomous stochastic differential equations, Electron. J. Differential Equations 2007 (2007), no. 117, $1-10$.

7. P.H. Bezandry and T. Diagana, Existence of quadratic-mean almost periodic solutions to some stochastic hyperbolic differential equations, Electron. J. Differential Equations 2009 (2009), no. 111, 1-14.

8. P.H. Bezandry and T. Diagana, Existence of square-mean almost periodic mild solutions to some nonautonomous stochastic second-order differential equations, Electron. J. Differential Equations 2010 (2010), no. 124, 1-25.

9. P.H. Bezandry and T. Diagana, Existence of $S^{2}$-almost periodic solutions to a class of nonautonomous stochastic evolution equations, Electron. J. Qual. Theory Differ. Equ. 2008 (2008), no. 35, 1-19.

10. J. Cao, Q. Yang, Z. Huang and Q. Liu, Asymptotically almost periodic solutions of stochastic functional differential equations, Appl. Math. Comput. 218 (2011), no. 5, 1499-1511

11. C. Cuevas and M. Pinto, Existence and uniqueness of pseudo almost periodic solutions of semilinear Cauchy problems with non dense domain, Nonlinear Anal. 45 (2001), no. 1, 73-83.

12. T. Diagana, Pseudo almost periodic solutions to some differential equations, Nonlinear Anal. 60 (2005), no. 7, 1277-1286.

13. T. Diagana, Existence and uniqueness of pseudo almost periodic solutions to some classes of partial evolution equations, Nonlinear Anal. 66 (2007), no. 2, 384-395.

14. T. Diagana and E.M. Hernández, Existence and uniqueness of pseudo almost periodic solutions to some abstract partial neutral functional differential equations and applications, J. Math. Anal. Appl. 327 (2007), no. 2, 776-791.

15. T. Diagana, Stepanov-like pseudo almost periodicity and its applications to some nonautonomous differential equations, Nonlinear Anal. 69 (2008), no. 12, 4277-4285.

16. T. Diagana, Stepanov-like pseudo almost periodic functions and their applications to differential equations, Commun. Math. Anal. 3 (2007), no. 1, 9-18.

17. T. Diagana, C.M. Mahop, G.M. N'Guérékata and B. Toni, Existence and uniqueness of pseudo almost periodic solutions to some classes of semilinear differential equations and applications, Nonlinear Anal. 64 (2006), no. 11, 2442-2453. 
18. T. Diagana and G.M. N'Guérékata, Pseudo almost periodic mild solutions to hyperbolic evolution equations in abstract intermediate Banach spaces, Appl. Anal. 85 (2006), no. 6-7, 769-780.

19. H.S. Ding, J. Liang, G.M. N'Guérékata and T.J. Xiao, Pseudo almost periodicity to some nonautonomous evolution equations with delay, Nonlinear Anal. 67 (2007), no. 5, 14121418.

20. H.S. Ding, J. Liang, G.M. N'Guérékata and T.J. Xiao, Mild pseudo almost periodic solutions of nonautonomous semilinear evolution equations, Math. Comput. Modelling 45 (2007), no. 5-6, 579-584.

21. A.Ya. Dorogovtsev and O.A. Ortega, On the existence of periodic solutions of a stochastic equation in a Hilbert space, Visnik Kiiv. Univ. Ser. Mat. Mekh. 115 (1988), no. 30, 21-30.

22. E. Hernández, Existence results for partial neutral integro-differential equations with unbounded delay, J. Math. Anal. Appl. 292 (2004), no. 1, 194-210.

23. E. Hernández and H.R. Henríquez, Existence results for partial neutral functional differential equations with unbounded delay, J. Math. Anal. Appl. 221 (1998), no. 2, 452-475.

24. E. Hernández and H.R. Henríquez, Pseudo almost periodic solutions for non-autonomous neutral differential equations with unbounded delay, Nonlinear Anal. RWA 9 (2008), no. 2, 430-437.

25. E. Hernández and H.L. Pelicer, Asymptotically almost periodic and almost periodic solutions for Partial neutral differential equations, Appl. Math. Lett. 18 (2005), no. 11, 1265-1272.

26. Z.R. Hu and Z. Jin, Stepanov-like peudo almost periodic mild solutions to perturbed nonautonomous evolution equations with infinite delay, Nonlinear Anal. 71 (2009), no. 11, 53815391.

27. Z. Huang and Q. Yang, Existence and exponential stability of almost periodic solution for stochastic cellular neural networks with delay, Chaos Solitons Fractals 42 (2009), no. 2, $773-780$.

28. A. Ichikawa, Stability of semilinear stochastic evolution equations, J. Math. Anal. Appl. 90 (1982), no. 1, 12-44.

29. D. Prato and C. Tudor, Periodic and almost periodic solutions for semilinear stochastic equations, Stoch. Anal. Appl. 13 (1995), no. 1, 13-33.

30. C. Tudor, Almost periodic solutions of affine stochastic evolution equations, Stoch. Stoch. Rep. 38 (1992), no. 4, 251-266.

31. C.A. Tudor and M. Tudor, Pseudo almost periodic solutions of some stochastic differential equations, Math. Rep. (Bucur.) 1(51) (1999), no. 2, 305-314.

32. J. Wu, Theory and Applications of Partial Functional-differential Equations, in: Appl. Math. Sci., vol. 119, Springer-Verlag, New York, 1996.

33. J. Wu and H. Xia, Rotating waves in neutral partial functional-differential equations, J. Dynam. Differential Equations 11 (1999), no. 2, 209-238.

34. J. Wu and H. Xia, Self-sustained oscillations in a ring array of coupled lossless transmission lines, J. Differential Equations 124 (1996), no. 1, 247-278.

35. C.Y. Zhang, Pseudo almost periodic solutions of some differential equations, J. Math. Anal. Appl. 181 (1994), no. 1, 62-76.

36. C.Y. Zhang, Pseudo almost periodic solutions of some differential equations II, J. Math. Anal. Appl. 192 (1995), no. 2, 543-561.

37. C.Y. Zhang, Integration of vector-valued pseudo almost periodic functions, Proc. Amer. Math. Soc. 121 (1994), no. 1, 167-174.

38. C.Y. Zhang, Pseudo Almost Periodic Functions and Their Applications, Thesis, the University of Western Ontario, 1992.

39. Z.H. Zhao, Y.K. Chang and W.S. Li, Asymptotically almost periodic, almost periodic and pseudo-almost periodic mild solutions for neutral differential equations, Nonlinear Anal. RWA 11 (2010), no. 4, 3037-3044. 
${ }^{1}$ Department of Mathematics, Hexi University, Zhangye, Gansu 734000, P.R. CHINA

E-mail address: yanzuomao@163.com

2 Department of Mathematics, Hexi University, Zhangye, Gansu 734000, P.R. CHINA

E-mail address: zh-hongwu@163.com 